\begin{tabular}{|r|l|}
\hline \multicolumn{2}{|c|}{ Statistica Sinica Preprint No: SS-2020-0415 } \\
\hline Title & $\begin{array}{l}\text { Tests of Unit Root Hypothesis With Heavy-Tailed } \\
\text { Heteroscedastic Noises }\end{array}$ \\
\hline Manuscript ID & SS-2020-0415 \\
\hline URL & http://www.stat.sinica.edu.tw/statistica/ \\
\hline DOI & $10.5705 /$ ss.202020.0415 \\
\hline Complete List of Authors & Rui She \\
\hline Corresponding Author & Rui She \\
\hline E-mail & rshe@swufe.edu.cn \\
\hline Notice: Accepted version subject to English editing.
\end{tabular}


Statistica Sinica

\title{
Tests of Unit Root Hypothesis with Heavy-tailed Heteroscedastic Noises
}

\author{
Rui She \\ Southwestern University of Finance and Economics
}

\begin{abstract}
This paper studies the unit-root testing with unspecified and heavy-tailed heteroscedastic noises. A new weighted least squares estimation (WLSE) is designed to be used in the Dickey-Fuller (DF) test, of which the asymptotic normality is verified. However, the performance of the DF test strongly relies on the estimation accuracy of the asymptotic variance, which is not stable for dependent time series. To overcome this issue, we develop two novel unit-root tests by applying the empirical likelihood technique to the WLSE score equation. It is shown that both of the empirical likelihood-based tests converge weakly to a chi-squared distribution with one degree of freedom. Furthermore, the limiting theory is extended to the weighted $M$-estimation score equation. In contrast to existing unit-root tests for heavy-tailed time series, the empirical likelihood tests do not involve any estimators of the unknown parameters or any restrictions on the tail index of noise, which is of more practical appealing, and thus can be widely used in finance and econometrics. Extensive simulation studies are conducted to examine the effectiveness of the proposed methods.
\end{abstract}

Key words and phrases: G/ARCH type noise; Heavy-tailed; Unit-root; Empirical likelihood. 


\section{Introduction}

Consider the following $\mathrm{AR}(1)$ model:

$$
y_{t}=\phi y_{t-1}+\varepsilon_{t}
$$

where the noise $\left\{\varepsilon_{t}\right\}$ is a sequence of stationary random variables. We are interested in detecting a possible unit root in model (1.1), namely, we test the null hypothesis $H_{0}: \phi=1$ versus the alternative $H_{1}:|\phi|<1$. There is an extensive and relatively complete literature on unit-root estimation and testing when $E \varepsilon_{t}^{2}$ is finite. When the noises $\left\{\varepsilon_{t}\right\}$ are independent and identically distributed (i.i.d.) random variables, based on the ordinary least squares estimator (LSE) of regression parameter, the classical Dickey-Fuller (DF) test and student $t$ test was proposed by Dickey and Fuller (1979) and Evans and Savin (1981), respectively. Phillips (1987) further studied these tests and established the corresponding limiting theory when the noise is strong-mixing. A concise review on this topic is refereed to Chan (2009).

In the past two decades, a growing number of the empirical studies have documented the heavy-tailed noise in financial markets. Koedijk and Kool (1992) studied the exchange rate returns for three East European currencies and found that their tail indices are smaller than 2. Francq and Zakoïan (2013) investigated nine major financial markets in the world and argued that the time series modelling driven by a heavy-tailed noise may be more appropriate to financial data analysis; see Rachev (2003) and She and Ling (2020), among many others. All previous evidences show 
that there is a practical and urgent need to study the heavy-tailed time series. Moreover, the unit-root detection for models with heavy-tailed innovations is of great importance and of more practical appealing.

However, when the noise is heavy-tailed (i.e., $E \varepsilon_{t}^{2}=\infty$ ), the unit-root inference is much more complicated and challenging, even for the i.i.d. case. For instance, Chan and Tran (1989) studied the DF test when $\varepsilon_{t}$ lies in the domain of attraction of a stable law with tail index $\alpha<2$, such that they have an infinite variance. They found that compared with the finite variance case, the limiting distributions of the classical DF tests are no longer pivotal since they depend on the unknown tail index of noise, which is very hard to estimate properly in practice (Resnick, 1997). To bypass the problem in heavy-tailed time series, one popular approach is to use the bootstrap or subsampling method to approximate the critical values. For example, Cavaliere, Georgiev and Taylor (2018) proposed a sieve wild bootstrap method to obtain the null distribution of augmented DF (ADF) test when the noise is a linear process driven by the i.i.d. heavy-tailed innovations; see Horváth and Kokoszka (2003) and Moreno and Romo (2012) for early work. Zhang and Chan (2020) extended the results in Cavaliere, Georgiev and Taylor (2018) to the case where the noise is from standard GARCH model, but the simulation results in their work indicate that the aforementioned wild bootstrap method can not deal with the heavy-tailed GARCH noises. Recently, Huang et al. (2020) proposed a novel empirical-likelihood-based 
method to construct a unified test for model (1.1) with the noise following from the standard $\operatorname{GARCH}(p, q)$ model, namely,

$$
\varepsilon_{t}=\eta_{t} h_{t}, \quad h_{t}^{2}=\omega+\sum_{i=1}^{p} \alpha_{i} \varepsilon_{t-i}^{2}+\sum_{j=1}^{q} \beta_{j} h_{t-j}^{2},
$$

where $\omega>0, \alpha_{i} \geq 0$ and $\beta_{j} \geq 0$. The core ingredient of their work is to bound the possible heavy tail of $h_{t}$ by some weighting or normalizing function so that the heavy tail effect in $h_{t}$ is eliminated, leading to the robustness of their test. By means of the empirical likelihood technique, their test also has the merit of getting rid of the estimation for the nuisance parameters. Nonetheless, the form of the normalizer they used may rely on the specific structure of $h_{t}$ in (1.2), making it not feasible to the cases without a priori knowledge on the structure of $h_{t}$. On top of that, the conditions imposed on the moment of $\eta_{t}$ and $h_{t}$ (e.g., $E \eta_{t}^{2}<\infty$ and $E h_{t}<\infty$ ) is relatively restrictive, which excludes the classical heavy-tailed i.i.d. case $\left(E \eta_{t}^{2}=\infty\right.$ and $h_{t}=1$ ). The heavy-tailed case with $E h_{t}=\infty$ is also indispensable, even under the condition $E \eta_{t}^{2}<\infty$, as illustrated by the domain $D_{2}$ in Figure 1. Therefore, these issues in Huang et al. (2020) motivate us to construct a unified unit-root test for model (1.1) or its extensions that is free of strong moment conditions on the noise and does not require a priori information on the structure of $h_{t}$.

In this paper, we study the unit-root process with unspecified and heavy-tailed heteroscedastic noises. To address the foregoing issues, a new weighted least squares estimation (WLSE) is proposed and embedded in the traditional DF test. We show 
that the derived DF-type test converges in distribution to a normal distribution under the null hypothesis and to negative infinity under the alternative. However, the performance of the new DF test is vulnerable to the estimation accuracy of the asymptotic variance that is not stable for strongly dependent time series. We thus develop two novel unit-root tests by applying empirical likelihood technique to the WLSE score equations. Both of the two empirical likelihood-based tests are shown to be asymptotically chi-squared with power approaching to one. The corresponding asymptotic theory is also extended to the general weighted $M$-estimation score equations. As expected, our unit-root tests get rid of the estimations of the regression parameters, the tail index of the noise, the structure of heteroscedasticity, and thus have a broader application in finance and econometrics. In addition, the proposed tests can be used in more general settings, such as the unit root with a constant term and the unit root in $\mathrm{AR}(\mathrm{r})$ model. A simulation study is conducted to demonstrate the performance of the proposed tests.

The rest of the paper is organized as follows. Section 2 gives a fundamental assumption and studies the DF-type test based on the new WLSE. Section 3 derives the asymptotic properties of the proposed unit-root tests via the standard empirical likelihood method and the adjusted empirical likelihood method with extensions to more general unit-root models in Section 4. The results of simulation studies and comparison with existing tests are summarized in Section 5. The technical proofs of 
main results are given in Supplementary Material.
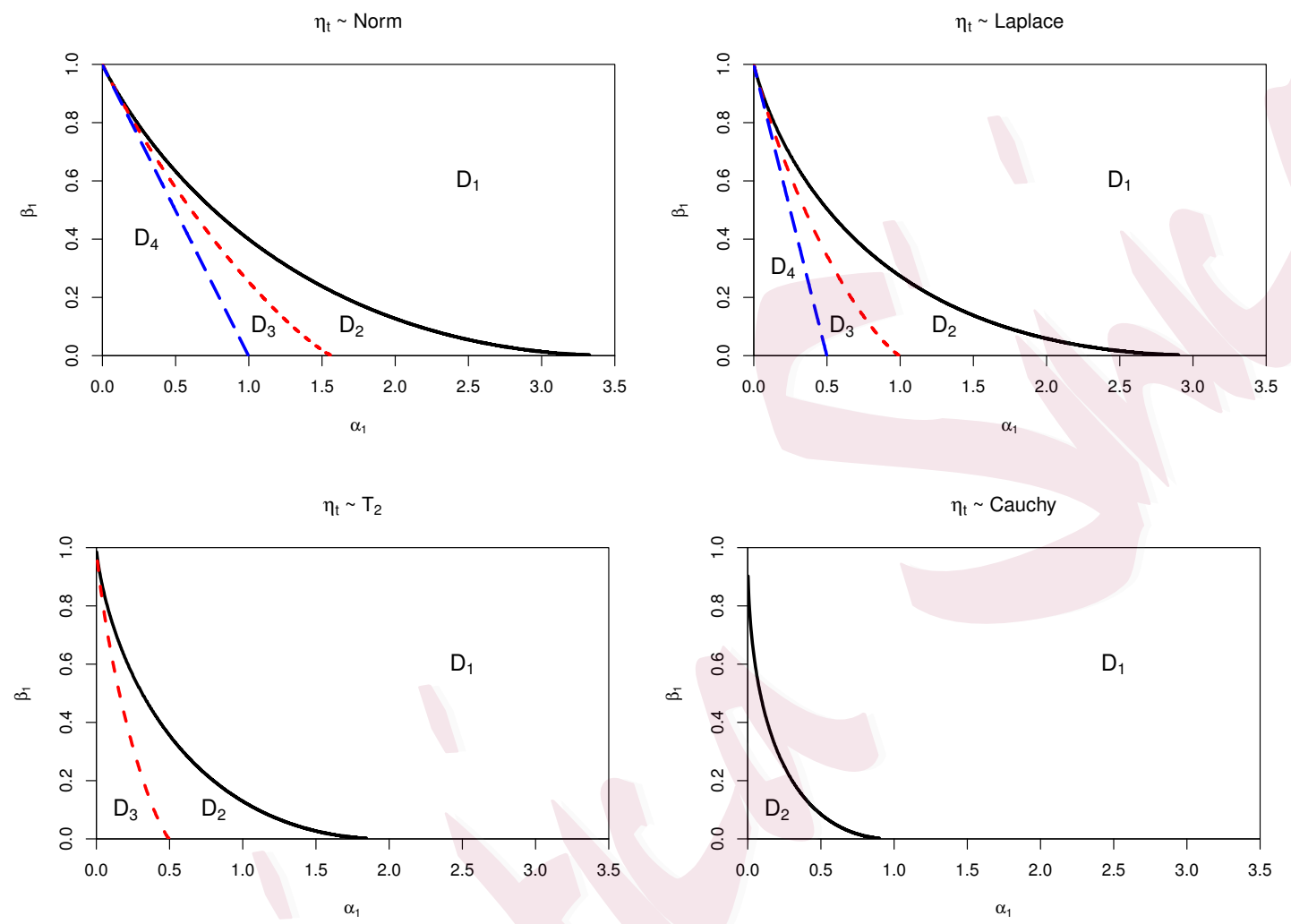

Figure 1: The regions of tail index $\alpha$ of $h_{t}$ in $\operatorname{GARCH}(1,1)$ model when $\eta_{t} \sim N(0,1)$, Laplace $(0,1)$, $t_{2}$ or Cauchy distribution, where $D_{1}$ means the domain of no stationary solution; $D_{2}$ means the domain of tail index $\alpha \in(0,1) ; D_{3}$ means the domain of tail index $\alpha \in(1,2) ; D_{4}$ means the domain of tail index $\alpha \in(2, \infty)$.

\section{Assumption and the WLSE}

\subsection{Assumption}


Throughout this paper, we focus on the noise satisfying the heteroscedastic form:

$$
\varepsilon_{t}=\eta_{t} h_{t} \quad \text { and } \quad h_{t}=h\left(\eta_{t-1}, \eta_{t-2}, \cdots\right)
$$

where the innovation $\left\{\eta_{t}\right\}$ is a sequence of i.i.d. symmetric random variables with $P\left(\eta_{t} \neq 0\right)>0$, and $h(\cdot)$ is a measurable positive function. Since the structure of $h_{t}$ is not specified, it is general enough and many popular G/ARCH type models are included in model (2.1), such as the absolute value GARCH model in Taylor (1986) and Schwert (1989), the nonlinear GARCH model in Engle (1990), the GJR model in Glosten, Jagannathan and Runkle (1993), the threshold GARCH model in Zakoïan (1994), the quadratic ARCH model in Sentana (1995), and the volatility switching GARCH model in Fornari and Mele (1997). We make a fundamental assumption on $\left\{\varepsilon_{t}\right\}$ as follows.

Assumption 2.1. There exists some strictly positive deterministic sequence $\left\{a_{n}\right\}$, such that, in the Skorohod space $\mathbb{D}[0,1]$ equipped with S-topology,

$$
S_{n}(\tau)=\frac{1}{a_{n}} \sum_{t=1}^{[n \tau]} \varepsilon_{t} \stackrel{w}{\longrightarrow} S(\tau),
$$

where $a_{n} \rightarrow \infty$ and $\int_{0}^{1} S^{2}(\tau) d \tau>0$ almost surely.

Assumption 2.1 is actually a very mild condition that allows for not only the finite variance case $(\alpha>2)$ but also the infinite variance case $(\alpha<2)$. To a better illustration, we now provide several examples and conditions under which 
Assumption 2.1 holds, especially for those commonly used in the literature and the model used in the simulation.

Note that the $S$-topology is a sequential topology on the Skorohod space $\mathbb{D}[0,1]$ proposed by Jakubowski (1997). By Proposition 3.1 and Theorem 3.5 in that paper, for Assumption 2.1 to be held, it is sufficient to show that

$$
\begin{aligned}
& \left(S_{n}\left(\tau_{1}\right), \cdots, S_{n}\left(\tau_{k}\right)\right) \stackrel{d}{\longrightarrow}\left(S\left(\tau_{1}\right), \cdots, S\left(\tau_{k}\right)\right), \forall k \in \mathbb{N} \text { and } \tau_{i} \in[0,1], \\
& \left\|S_{n}\right\|=O_{p}(1), \text { and for any } a<b, \text { we have } N^{a, b}\left(S_{n}\right)=O_{p}(1),
\end{aligned}
$$

where $\left\|S_{n}\right\|=\sup _{\tau \in[0,1]}\left|S_{n}(\tau)\right|$ and $N^{a, b}\left(S_{n}\right)$ is the usual number of up-crossing defined by the relation: $N^{a, b}\left(S_{n}\right) \geq l$ if and only if there exist numbers $0 \leq \tau_{1}<$ $\tau_{2}<\cdots<\tau_{2 l-1}<\tau_{2 l} \leq 1$ such that $S_{n}\left(\tau_{2 i}\right)>b$ and $S_{n}\left(\tau_{2 i-1}\right)<a$ for all $i=$ $1, \cdots, l$. Although the weak convergence in $S$-topology is much weaker than that in $J_{1}$-topology (or uniform topology), it has been proved that the well-known a.s. Skorohod representation theorem still holds, see Jakubowski (1997) for details. Thus, the $S$-topology can be widely used in many scenarios, especially for heavy-tailed data.

Remark 2.1. The convergence in (2.2) is well known as the convergence of finitedimension distribution and has been evidenced in many G/ARCH type processes. For example, consider one representative class of G-GARCH process in Zhang and Ling (2015) with

$$
h_{t}^{2}=\omega+c\left(\eta_{t-1}\right) h_{t-1}^{2}
$$


where $\omega>0$ and $c(\cdot)$ is a non-negative function with $c(0)<1$. Zhang and Ling (2015) have shown that there exists a unique $\alpha \in\left(0,2 k_{0}\right]$ such that $E\left(c\left(\eta_{t}\right)\right)^{\alpha / 2}=1$ and the noise $\varepsilon_{t}$ is a regular variation with tail index $\alpha$ (i.e., $P\left(\left|\varepsilon_{t}\right|>x\right) \sim x^{-\alpha}$ ), under the following conditions:

(a). $E \log \left(c\left(\eta_{t}\right)\right)<0$;

(b). There exists a $k_{0}>0$ such that $E\left(c\left(\eta_{t}\right)\right)^{k_{0}} \geq 1$ and $E\left[\left(c\left(\eta_{t}\right)\right)^{k_{0}} \log ^{+}\left(c\left(\eta_{t}\right)\right)\right]<\infty$, and $E\left(\left|\eta_{t}\right|^{2 k_{0}}\right)<\infty$, where $\log ^{+}(x)=\max \{0, \log (x)\}$;

(c). The density $f(x)$ of $\eta_{t}$ is positive in the neighbourhood zero.

Furthermore, by similar arguments in the proof of Theorem 2.1 in Chan and Zhang (2010), it is straightforward to get the condition (2.2), in which $a_{n}=\sqrt{n}$ for $\alpha>2$ (light-tail) and $a_{n}=n^{1 / \alpha}$ for $\alpha<2$ (heavy-tail).

Remark 2.2. On the other hand, one can easily show that the condition (2.3) is satisfied for the two foregoing cases. The first one is the case when the tail index $\alpha>2$ and $a_{n}=\sqrt{n}$. In this case, since $S_{n}(\tau)$ is martingale and by the Doob's inequality, it follows that, for any $M>0$,

$$
P\left(\left\|S_{n}\right\|>M\right) \leq 3 M^{-1} \sup _{\tau \in[0,1]} E\left|S_{n}(\tau)\right|, \quad E N^{a, b}\left(S_{n}\right) \leq \frac{1}{b-a}\left(|a|+\sup _{\tau \in[0,1]} E\left|S_{n}(\tau)\right|\right) .
$$

Then the condition (2.3) holds from $\sup _{n} \sup _{\tau \in[0,1]} E\left|S_{n}(\tau)\right| \leq\left(E \varepsilon_{t}^{2}\right)^{1 / 2}<\infty$. The 
other case is the one with the tail index $\alpha<2$ and $a_{n}=n^{1 / \alpha}$. In this case, rewrite

$$
S_{n}(\tau)=\sum_{t=1}^{[n \tau]} \varepsilon_{t} 1_{\left(\left|\varepsilon_{t}\right|<a_{n}\right)} / a_{n}+\sum_{t=1}^{[n \tau]} \varepsilon_{t} 1_{\left(\left|\varepsilon_{t}\right| \geq a_{n}\right)} / a_{n}=S_{n 1}(\tau)+S_{n 2}(\tau)
$$

Notice that $S_{n 1}(\tau)$ is still martingale and by Karamata's theorem, we have

$$
\sup _{\tau \in[0,1]} E S_{n 1}^{2}(\tau)=\frac{n E \varepsilon_{t}^{2} 1_{\left(\left|\varepsilon_{t}\right|<a_{n}\right)}}{a_{n}^{2}} \longrightarrow \frac{\alpha}{2-\alpha}, \text { as } n \rightarrow \infty
$$

Then, the condition (2.3) holds for $S_{n 1}(\tau)$. Furthermore, choose $\rho \in(0, \alpha \wedge 1)$, then by Karamata's theorem again, we have

$$
E\left\|S_{n 2}\right\|^{\rho} \leq \frac{n E\left|\varepsilon_{t}\right|^{\rho} 1_{\left(\left|\varepsilon_{t}\right| \geq a_{n}\right)}}{a_{n}^{\rho}} \longrightarrow \frac{\alpha}{\alpha-\rho}, \text { as } n \rightarrow \infty
$$

which implies that $\left\|S_{n 2}\right\|=O_{p}(1)$. For $N^{a, b}\left(S_{n 2}\right)$, we only need to notice that $N^{a, b}\left(S_{n 2}\right) \leq \sum_{t=1}^{n} 1_{\left(\left|\varepsilon_{t}\right| \geq a_{n}\right)}$, then $\lim \sup _{n} E N^{a, b}\left(S_{n 2}\right) \leq \lim \sup _{n} n P\left(\left|\varepsilon_{t}\right| \geq a_{n}\right)<\infty$ Thus, (2.3) holds for $S_{n 2}(\tau)$. As a result, the condition (2.3) holds for $S_{n}(\tau)=$ $S_{n 1}(\tau)+S_{n 2}(\tau)$

\subsection{The WLSE approach}

Now, we investigate the estimation of $\phi$. The ordinary LSE is defined as

$$
\hat{\phi}_{l s e}=\arg \min \sum_{t=1}^{n}\left(y_{t}-\phi y_{t-1}\right)^{2}=\frac{\sum_{t=1}^{n} y_{t} y_{t-1}}{\sum_{t=1}^{n} y_{t}^{2}} .
$$

Conventionally, when $\phi=1$ (i.e., under $H_{0}$ ), Assumption 2.1 may imply that

$$
n\left(\hat{\phi}_{l s e}-1\right)=n \frac{\sum_{t=1}^{n} \varepsilon_{t} y_{t-1}}{\sum_{t=1}^{n} y_{t}^{2}} \stackrel{d}{\longrightarrow} \frac{\int_{0}^{1} S^{-}(\tau) d S(\tau)}{\int_{0}^{1} S^{2}(\tau) d \tau}
$$


where $S^{-}(\tau)$ denotes the left-hand limit of $S(\tau)$. When the variance of $\varepsilon_{t}$ is infinite, $S(\tau)$ is always a stable process with tail index smaller than 2, as in Chan and Zhang (2010) and the references therein. In this case, the above limiting distribution is not pivotal and the existing bootstrap methods are very sensitive to the structure and tail index of $\varepsilon_{t}$ (Cavaliere, Georgiev and Taylor, 2018; Zhang and Chan, 2020). It seems infeasible to use a unified bootstrap method to deal with this issue.

Inspired by the main idea of Chan, Li and Peng (2012) and Huang et al. (2020), we define the weighted least squares estimator (WLSE) as

$$
\hat{\phi}_{w l s e}=\arg \min \sum_{t=1}^{n} \frac{\left(y_{t}-\phi y_{t-1}\right)^{2}}{\left(1+y_{t-1}^{2}\right)^{1 / 2}\left[1+\left(\Delta y_{t}\right)^{2}\right]^{1 / 2}},
$$

where $\Delta y_{t}=y_{t}-y_{t-1}$. Then, under the null hypothesis, it is easy to get that

$$
\sum_{t=1}^{n} \frac{y_{t-1}^{2}}{\left(1+y_{t-1}^{2}\right)^{1 / 2}\left[1+\left(\Delta y_{t}\right)^{2}\right]^{1 / 2}}\left(\hat{\phi}_{w l s e}-1\right)=\sum_{t=1}^{n} \frac{y_{t-1} \varepsilon_{t}}{\left(1+y_{t-1}^{2}\right)^{1 / 2}\left[1+\left(\Delta y_{t}\right)^{2}\right]^{1 / 2}}
$$

Thus, we can derive the DF-type test statistic:

$$
T_{n}=n^{-1 / 2} \sum_{t=1}^{n} \frac{y_{t-1}^{2}}{\left(1+y_{t-1}^{2}\right)^{1 / 2}\left[1+\left(\Delta y_{t}\right)^{2}\right]^{1 / 2}}\left(\hat{\phi}_{w l s e}-1\right) .
$$

The asymptotic properties of $T_{n}$ are given in Theorem 2.1 below.

Theorem 2.1. Suppose that Assumption 2.1 holds. Under $H_{0}$, it follows that

$$
T_{n} \stackrel{d}{\longrightarrow} N\left(0, \sigma^{2}\right)
$$

where $\sigma^{2}=E\left[\varepsilon_{t}^{2} /\left(1+\varepsilon_{t}^{2}\right)\right]$. Under $H_{1}$, it follows that $T_{n} \stackrel{p}{\longrightarrow}-\infty$. 
Remark 2.3. In practice, we can replace $\sigma^{2}$ by $\hat{\sigma}^{2}=n^{-1} \sum_{t=1}^{n}\left(\Delta y_{t}\right)^{2} /\left[1+\left(\Delta y_{t}\right)^{2}\right]$ and develop the WLSE-based test as

$$
\tilde{T}_{n}=T_{n} / \hat{\sigma}
$$

Since $\hat{\sigma}$ is a consistent estimator of $\sigma$ under $H_{0}$ by (2.1) and the ergodic theorem, it is obvious that $\tilde{T}_{n}$ converges in distribution to $N(0,1)$. Meanwhile, by the fact that $\hat{\sigma}$ is bounded by 1 , we still have $\tilde{T}_{n} \stackrel{p}{\longrightarrow}-\infty$ under $H_{1}$. Then, at the given significant level $\alpha$, the null hypothesis should be rejected when $\tilde{T}_{n}<u_{\alpha}$, where $u_{\alpha}$ denotes the $\alpha$-th quantile of the standard normal distribution. Under this criteria, the power is approaching to one as $n \rightarrow \infty$.

However, it is well-known that the estimation of the asymptotic variance is not always stable, especially for strongly dependent time series or small sample size. As a result, the WLSE-based test $\tilde{T}_{n}$ may suffer from a serious size distortion, as shown in Section 5. In the next section, we attempt to bypass the estimation of nuisance parameters by means of the empirical likelihood technique in Owen (2001), which has been found very useful in many scientific fields.

\section{Empirical Likelihood Methods}

\subsection{Empirical likelihood test}

Recall that the proposed WLSE is based on the core idea that, under the null hypothesis, the heavy-tailed term $\varepsilon_{t}$ (i.e., $\left.\Delta y_{t}\right)$ can be bounded by $\left[1+\left(\Delta y_{t}\right)^{2}\right]^{1 / 2}$, 
while $y_{t-1}$ can be bounded by $\left(1+y_{t-1}^{2}\right)^{1 / 2}$. Thus, we consider the score function:

$$
Z_{t}(\phi)=\frac{y_{t-1}\left(y_{t}-\phi y_{t-1}\right)}{\left(1+y_{t-1}^{2}\right)^{1 / 2}\left[1+\left(y_{t}-\phi y_{t-1}\right)^{2}\right]^{1 / 2}}
$$

The empirical likelihood function is given by

$$
L(\phi)=\sup \left\{\prod_{t=1}^{n}\left(n p_{t}\right): \sum_{t=1}^{n} p_{t}=1, \sum_{t=1}^{n} p_{t} Z_{t}(\phi)=0, p_{t}>0, t=1, \cdots, n\right\} .
$$

Using the Lagrange multiplier technique, we can show that

$$
L(\phi)=\prod_{t=1}^{n} \frac{1}{1+\lambda Z_{t}(\phi)}
$$

where the Lagrange multiplier $\lambda$ is the solution of

$$
\sum_{t=1}^{n} \frac{Z_{t}(\phi)}{1+\lambda Z_{t}(\phi)}=0
$$

At the same time, the empirical log-likelihood ratio is

$$
l(\phi)=-2 \log L(\phi)=2 \sum_{t=1}^{n} \log \left[1+\lambda Z_{t}(\phi)\right]
$$

Now, we give the asymptotic results for $l(\phi)$ as follows.

Theorem 3.1. Suppose that Assumption 2.1 holds. Under $H_{0}$, it follows that

$$
l(1) \stackrel{d}{\longrightarrow} \chi_{1}^{2},
$$

as $n \rightarrow \infty$. Under $H_{1}$, we have $l(1) \stackrel{p}{\longrightarrow} \infty$ and $l(\phi) \stackrel{d}{\longrightarrow} \chi_{1}^{2}$, as $n \rightarrow \infty$. 
According to Theorem 3.1, we reject the null hypothesis at the significant level $\alpha$ if $l(1)>\chi_{1,1-\alpha}^{2}$, where $\chi_{1,1-\alpha}^{2}$ denotes the $(1-\alpha)$-th quantile of a chi-squared distribution with one degree of freedom. After rejecting $H_{0}$, Theorem 3.1 implies that the confidence interval for $\phi$ at level $1-\alpha$ can be constructed as

$$
I_{1-\alpha}=\left\{\phi: l(\phi)<\chi_{1,1-\alpha}^{2}\right\}
$$

Remark 3.1. It is worth mentioning that the new empirical likelihood test based on the score function $Z_{t}(\phi)$ in $(3.1)$ is essentially distinct from the empirical likelihood test (ELT) proposed by Huang et al. (2020) in both model settings and methodology. In our settings, under the null,

$$
Z_{t}(1)=\frac{y_{t-1} \Delta y_{t}}{\left(1+y_{t-1}^{2}\right)^{1 / 2}\left[1+\left(\Delta y_{t}\right)^{2}\right]^{1 / 2}}
$$

Since $\Delta y_{t}=\varepsilon_{t}$ must be bounded by the normalizer $\left[1+\left(\Delta y_{t}\right)^{2}\right]^{1 / 2}$, all the heavy-tailed effects appeared in the noise $\varepsilon_{t}$ are cancelled out. As a result, our method does not rely on any specific form of $h(\cdot)$ and no moment condition on $h_{t}$ or $\eta_{t}$ is required, making it applicable to many popular G/ARCH type models, such as standard GARCH, nonlinear GARCH and GJR, among many others. By contrast, Huang et al. (2020) mainly considered the noise $\varepsilon_{t}$ satisfying the standard $\operatorname{GARCH}(p, q)$ model as in $(1.2)$ and their ELT method depends on the functional of $h(\cdot)$. For instance, when $q=0$ 
(ARCH model), under the null, they use the following score equation:

$$
\begin{aligned}
Y_{t}(1) & =\frac{y_{t-1} \Delta y_{t}}{\left(1+y_{t-1}^{2}\right)^{1 / 2}\left[1+\sum_{k=1}^{m}\left(\Delta y_{t-k}\right)^{2}\right]^{1 / 2}} \\
& =\eta_{t} \times \frac{y_{t-1} h_{t}}{\left(1+y_{t-1}^{2}\right)^{1 / 2}\left[1+\sum_{k=1}^{m}\left(\Delta y_{t-k}\right)^{2}\right]^{1 / 2}},
\end{aligned}
$$

where $m$ is chosen to be larger than $p$ to guarantee the inequality

$$
h_{t}^{2} \leq \max \left\{\omega, \alpha_{1}, \cdots, \alpha_{p}\right\}\left[1+\sum_{k=1}^{m}\left(\Delta y_{t-k}\right)^{2}\right]
$$

Therefore, a priori information on the model structure $h(\cdot)$ is indispensable, which, however, is typically unknown in practice, especially when one is at the stage of checking the stationarity of time series. On the other hand, the normalizer $[1+$ $\left.\sum_{k=1}^{m}\left(\Delta y_{t-k}\right)^{2}\right]^{1 / 2}$ in the denominator of $Y_{t}(1)$ is only able to remove the heavytailed effect in $h_{t}$, resulting in the inefficiency for the case with $E \eta_{t}^{2}=\infty$ as shown in Section 5. More details, for example, can be found in Figure 1, in which Huang et al. (2020) may not be able to handle the domain $D_{2}$.

\subsection{Adjusted empirical likelihood test}

In order to improve the size performance of the proposed empirical likelihood test, we further consider the adjusted empirical likelihood approach proposed by Chen, Variyath and Abraham (2008). Define the additional term:

$$
Z_{n+1}(\phi)=-b_{n} n^{-1} \sum_{t=1}^{n} Z_{t}(\phi)
$$


where $Z_{t}(\phi)$ is defined in (3.1) and $b_{n}$ is some positive constant. Then, the adjusted empirical likelihood function is defined as

$$
L^{a}(\phi)=\sup \left\{\prod_{t=1}^{n+1}(n+1) p_{t}: p_{t}>0, t=1, \cdots, n+1 ; \sum_{t=1}^{n+1} p_{t}=1, \sum_{t=1}^{n+1} p_{t} Z_{t}(\phi)=0\right\}
$$

Similarly, the corresponding adjusted empirical log-likelihood ratio is

$$
l^{a}(\phi)=-2 \log L^{a}(\phi)=2 \sum_{t=1}^{n+1} \log \left[1+\lambda Z_{t}(\phi)\right]
$$

where the Lagrange multiplier $\lambda$ is the solution of

$$
\sum_{t=1}^{n+1} \frac{Z_{t}(\phi)}{1+\lambda Z_{t}(\phi)}=0
$$

Then, the limiting theory of $l^{a}(\phi)$ can be derived as follows.

Theorem 3.2. Suppose that Assumption 2.1 holds and $b_{n} / n+1 / b_{n}=o(1)$. Under $H_{0}$, it follows that

$$
l^{a}(1) \stackrel{d}{\longrightarrow} \chi_{1}^{2}
$$

as $n \rightarrow \infty$. Under $H_{1}$, we have $l^{a}(1) \stackrel{p}{\longrightarrow} \infty$ and $l^{a}(\phi) \stackrel{d}{\longrightarrow} \chi_{1}^{2}$, as $n \rightarrow \infty$.

Theorem 3.2 shows that we need to reject the null hypothesis at the significant level $\alpha$ if $l^{a}(1)>\chi_{1,1-\alpha}^{2}$ and the confidence interval of $\phi$ at level $1-\alpha$ is constructed as $I_{1-\alpha}^{a}=\left\{\phi: l^{a}(\phi)<\chi_{1,1-\alpha}^{2}\right\}$.

Remark 3.2. Here, we simply point out the difference between two empirical likelihood methods based on $l(\phi)$ and $l^{a}(\phi)$. By the definition of $L(\phi)$, we can see that 
the necessary and sufficient condition for its existence is that the original point is an interior point of the convex hull of $\left\{Z_{t}(\phi), t \leq n\right\}$. Under some moment and dependent assumptions, this condition can hold with probability to 1 as $n \rightarrow \infty$ (Owen, 2001). However, for general time series or in small sample size, this may be a serious limitation (Chen, Variyath and Abraham, 2008). Thus, the adjusted term $Z_{n+1}(\phi)$ is used to ensure that the original point is an interior point of the convex hull of $\left\{Z_{t}(\phi), t \leq n+1\right\}$ such that $L^{a}(\phi)$ is well-defined. As will be seen in our simulation later, $l^{a}(1)$ has a better size performance than $l(1)$. Meanwhile, $b_{n}$ can be chosen to $\max \{1, \log (n) / 2\}$ recommended by Chen, Variyath and Abraham (2008). For more discussions on the two empirical likelihood tests, we refer to Zheng and Yu (2013).

In the final of this section, we are ready to extend the score function $Z_{t}(\phi)$ to a more general form

$$
Z_{t}(\phi)=\frac{y_{t-1}}{\left(1+y_{t-1}^{2}\right)^{1 / 2}} \rho\left(y_{t}-\phi y_{t-1}\right), \quad t=1, \cdots, n
$$

and $Z_{n+1}(\phi)=-b_{n} n^{-1} \sum_{t=1}^{n} Z_{t}(\phi)$, where $\rho(x)$ is a function on the real line. In a similar proof to Theorems 3.1-3.2, it is not hard to get the following corollary.

Corollary 3.1. Suppose that Assumption 2.1 holds and $b_{n} / n+1 / b_{n}=o(1)$. If $\rho(x)$ is a bounded odd monotonic function, and one of the following conditions holds:

1. $\rho(x)$ is strictly monotonic;

2. $\rho(x) \neq \rho(y), \forall x y<0$ and the density of $\eta_{t}$ is positive in neighbourhood of zero. 
Then, all the limiting results in Theorems 3.1-3.2 still hold.

Remark 3.3. The traditional $M$-estimator $\hat{\phi}_{M}$ of $\phi$ is the solution of the equation:

$$
\sum_{t=1}^{n} y_{t-1} \rho\left(y_{t}-\phi y_{t-1}\right)=0
$$

where $\rho(x)$ is typically the first derivative of some loss function. In this case, the statistical inference is based on the asymptotic property of $\hat{\phi}_{M}$. To guarantee the derived DF-type test to be asymptotically Gaussian under $H_{0}$, the additional continuous conditions for $\rho(x)$ are often needed (Knight, 1991; Shin and So, 1999; Samarakoon and Knight, 2009). However, the proposed unit-root tests do not rely on any estimator of $\phi$ or need any continuous assumption for $\rho(\cdot)$. Thus, many widely-used functions are incorporated in this framework, such as the Huber function $\rho(x)=\min [c, \max (-c, x)]$ and the sign function $\rho(x)=1_{(x>0)}-1_{(x<0)}$.

\section{Extensions to other models}

In this section, we further generalize the proposed empirical likelihood method to other unit-root models. We first study the unit-root model with a constant term, namely,

$$
y_{t}=\mu+\phi y_{t-1}+\varepsilon_{t},
$$

where $\mu$ is a constant and $\varepsilon_{t}$ satisfies the heteroscedastic form (2.1). Recall that the 
LSE of the parameters $(\phi, \mu)$ is the solution of the equations:

$$
\sum_{t=1}^{n} \varepsilon_{t}(\phi, \mu)=0 \text { and } \sum_{t=1}^{n} y_{t-1} \varepsilon_{t}(\phi, \mu)=0
$$

where $\varepsilon_{t}(\phi, \mu)=y_{t}-\mu-\phi y_{t-1}$. Then, it is natural to consider the weighted LSE score equations $\mathbf{Z}_{t}(\phi, \mu)=\left(Z_{t, 1}(\phi, \mu), Z_{t, 2}(\phi, \mu)\right)^{\prime}$ with

$$
Z_{t, 1}(\phi, \mu)=\frac{\varepsilon_{t}(\phi, \mu)}{\left[1+\varepsilon_{t}^{2}(\phi, \mu)\right]^{1 / 2}} \text { and } Z_{t, 2}(\phi, \mu)=\frac{y_{t-1}}{\left(1+y_{t-1}^{2}\right)^{1 / 2}} Z_{t, 1}(\phi, \mu)
$$

Under $H_{0}$, since $\left|y_{n}\right| /\left(1+y_{n}^{2}\right)^{1 / 2} \stackrel{p}{\longrightarrow} 1$, it is not hard to show that

$$
n^{-1 / 2} \sum_{t=1}^{n} Z_{t, i}\left(1, \mu_{0}\right) \stackrel{d}{\longrightarrow} N\left(0, \sigma^{2}\right)
$$

for $i=1,2$, where $\mu_{0}$ is the true parameter and $\sigma^{2}$ is defined in Theorem 2.1. Meanwhile, $n^{-1 / 2} \sum_{t=1}^{n} Z_{t, 2}\left(1, \mu_{0}\right)$ is asymptotically equivalent to $n^{-1 / 2} \sum_{t=1}^{n} Z_{t, 1}\left(1, \mu_{0}\right)$, which implies that both of them are not bivariate normal. To overcome this degenerate issue, similar to Li, Chan and Peng (2014) and Huang et al. (2020), we add some independent samples into the score equations, specifically, define $\tilde{\mathbf{Z}}_{t}(\phi, \mu)=$ $\left(\tilde{Z}_{t, 1}(\phi, \mu), \tilde{Z}_{t, 2}(\phi, \mu)\right)^{\prime}$ with

$$
\begin{aligned}
& \tilde{Z}_{t, 1}(\phi, \mu)=\frac{\varepsilon_{t}(\phi, \mu)}{\left[1+\varepsilon_{t}^{2}(\phi, \mu)\right]^{1 / 2}} \\
& \tilde{Z}_{t, 2}(\phi, \mu)=\frac{y_{t-1}}{\left(1+y_{t-1}^{2}\right)^{\delta}} \tilde{Z}_{t, 1}(\phi, \mu)+w_{t},
\end{aligned}
$$

where the constant $\delta>1 / 2$ and $w_{t}$ is a sequence of i.i.d. random variables with $P\left(w_{t}= \pm 1\right)=1 / 2$. As suggested by Li, Chan and Peng (2014), $\delta$ is usually set to 
be 0.75 . Then, the associated empirical likelihood function is given by

$$
\tilde{L}(\phi, \mu)=\sup \left\{\prod_{t=1}^{n}\left(n p_{t}\right): p_{t}>0, \sum_{t=1}^{n} p_{t}=1, \sum_{t=1}^{n} p_{t} \tilde{\mathbf{Z}}_{t}(\phi, \mu)=\mathbf{0}\right\}
$$

Since the true parameter $\mu_{0}$ is unknown, we need to consider the profile empirical likelihood function $\tilde{L}(\phi)=\max _{\mu} \tilde{L}(\phi, \mu)$ and put $\tilde{l}(\phi)=-2 \log (\tilde{L}(\phi))$. The following theorem gives its limiting property.

Theorem 4.1. Suppose that Assumption 2.1 holds and $a_{n} / n \rightarrow c \in[0, \infty]$. Then, under $H_{0}$, it follows that $\tilde{l}(1) \stackrel{d}{\longrightarrow} \chi_{1}^{2}$, as $n \rightarrow \infty$. Furthermore, under $H_{1}$, it follows that $\tilde{l}(1) \stackrel{p}{\longrightarrow} \infty$.

Now, we investigate a more complicated unit-root $\mathrm{AR}(r)$ model with a constant term, namely,

$$
y_{t}=\mu+\phi y_{t-1}+\sum_{j=1}^{r} \phi_{j} \Delta y_{t-j}+\varepsilon_{t}, \quad r \geq 1
$$

Denote $\boldsymbol{\theta}=\left(\mu, \phi_{1}, \cdots, \phi_{r}\right)^{\prime}$ and $\varepsilon_{t}(\phi, \boldsymbol{\theta})=y_{t}-\mu-\phi y_{t-1}-\sum_{j=1}^{r} \phi_{j} \Delta y_{t-j}$. Note that, under the null hypothesis, the term $\Delta y_{t-j}$ is also likely to be heavy-tailed and thus the score equations are modified as follows:

$$
\begin{aligned}
\bar{Z}_{t, 1}(\phi, \boldsymbol{\theta}) & =\frac{\varepsilon_{t}(\phi, \boldsymbol{\theta})}{\left[1+\sum_{j=1}^{r}\left(\Delta y_{t-j}\right)^{2}\right]^{3 / 2}\left[1+\varepsilon_{t}^{2}(\phi, \boldsymbol{\theta})\right]^{1 / 2}} \\
\bar{Z}_{t, 2}(\phi, \boldsymbol{\theta}) & =\frac{y_{t-1}}{\left(1+y_{t-1}^{2}\right)^{\delta}} \bar{Z}_{t, 1}(\phi, \boldsymbol{\theta})+w_{t} \\
\bar{Z}_{t, 2+j}(\phi, \boldsymbol{\theta}) & =\frac{\Delta y_{t-j}}{\left[1+\left(\Delta y_{t-j}\right)^{2}\right]^{1 / 2}} \bar{Z}_{t, 1}(\phi, \boldsymbol{\theta}), \text { for } j=1, \cdots, r
\end{aligned}
$$


where the additional term $\left[1+\sum_{j=1}^{r}\left(\Delta y_{t-j}\right)^{2}\right]^{3 / 2}$ in the denominator is used to bound $\partial^{k} \bar{Z}_{t, 1}(\phi, \boldsymbol{\theta}) / \partial \boldsymbol{\theta}^{k}$ for $k=1,2,3$. Let $\overline{\mathbf{Z}}_{t}(\phi, \boldsymbol{\theta})=\left(\bar{Z}_{t, 1}(\phi, \boldsymbol{\theta}), \bar{Z}_{t, 2}(\phi, \boldsymbol{\theta}), \cdots, \bar{Z}_{t, 2+r}(\phi, \boldsymbol{\theta})\right)^{\prime}$ and the empirical likelihood function is

$$
\bar{L}(\phi, \boldsymbol{\theta})=\sup \left\{\prod_{t=1}^{n}\left(n p_{t}\right): p_{t}>0, \sum_{t=1}^{n} p_{t}=1, \sum_{t=1}^{n} p_{t} \overline{\mathbf{Z}}_{t}(\phi, \boldsymbol{\theta})=\mathbf{0}\right\}
$$

Similarly, define $\bar{L}(\phi)=\max _{\boldsymbol{\theta}} \bar{L}(\phi, \boldsymbol{\theta})$ and $\bar{l}(\phi)=-2 \log (\bar{L}(\phi))$. Notice that $\Delta y_{t}$ is a linear process with respect to the noise $\varepsilon_{t}$. Hence, before showing its limiting property, we need one counterpart of Assumption 2.1 as follows.

Assumption 4.1. There exists some deterministic sequence $\left\{\bar{a}_{n}\right\}$, such that

$$
\bar{S}_{n}(\tau)=\frac{1}{\bar{a}_{n}} \sum_{t=1}^{[n \tau]} u_{t} \stackrel{w}{\longrightarrow} \bar{S}(\tau)
$$

where $\bar{a}_{n} \rightarrow \infty$ and $u_{t}=\sum_{l=0}^{\infty} \rho_{l} \varepsilon_{t-l}$ with $\rho_{l}=O\left(\rho^{l}\right)$ for some $\rho \in(0,1)$.

Remark 4.1. When $\varepsilon_{t}$ is a sequence of i.i.d. heavy-tailed noises, Avram and Taqqu (1992) has shown that, under the classical $J_{1}$ topology, Assumption 4.1 holds if and if only the linear process $\left\{u_{t}\right\}$ is independent. Assumption 4.1, though seems to be unreasonable under the $J_{1}$ topology, does make sense under the $S$-topology. Indeed, one can easily derive Assumption 4.1 from Assumption 2.1 using the sufficient conditions in (2.2) and (2.3), if the additional condition holds:

$$
\lim _{H \rightarrow \infty} \limsup _{n \rightarrow \infty} P\left(\sup _{0 \leq \tau \leq 1}\left|a_{n}^{-1} \sum_{t=1}^{[n \tau]} u_{t, H}\right|>\eta\right)=0
$$


where $\eta$ is any positive number and $u_{t, H}=\sum_{l=H+1}^{\infty} \rho_{l} \varepsilon_{t-l}$. In this case, $\bar{S}(\tau)=$ $\left(\sum_{l=0}^{\infty} \rho_{l}\right) S(\tau)$ and $\bar{a}_{n}=a_{n}$; see Zhang, Sin and Ling (2015) for some examples.

Theorem 4.2. Suppose that Assumption 4.1 holds and $\bar{a}_{n} / n \rightarrow c \in[0, \infty]$. Then, under $H_{0}$, if $\left\{\Delta y_{t}\right\}$ is strictly stationary, then it follows that $\bar{l}(1) \stackrel{d}{\longrightarrow} \chi_{1}^{2}$, as $n \rightarrow \infty$. Furthermore, under $H_{1}$, if $\left\{y_{t}\right\}$ is strictly stationary, then it follows that $\bar{l}(1) \stackrel{p}{\longrightarrow} \infty$.

Theorems 4.1-4.2 show that the proposed profile empirical likelihood tests are still asymptotically chi-squared, even in more complicated unit root models (4.1) and (4.3). In general, if time series $\left\{y_{t}\right\}$ is generated from the linear model:

$$
y_{t}=\sum_{i=1}^{k} \gamma_{i} f_{i}(t)+\phi y_{t-1}+\sum_{j=1}^{r} \phi_{j} \Delta y_{t-j}+\varepsilon_{t}+\sum_{l=1}^{m} \rho_{l} \varepsilon_{t-l},
$$

where $\left\{f_{i}(t)\right\}$ are time trend functions. Let $\boldsymbol{\theta}=\left(\gamma_{i}, \cdots, \phi_{j}, \cdots, \rho_{l}, \cdots\right)^{\prime}$ and define

$$
\varepsilon_{t}(\phi, \boldsymbol{\theta})=y_{t}-\sum_{i=1}^{k} \gamma_{i} f_{i}(t)-\phi y_{t-1}-\sum_{j=1}^{r} \phi_{j} \Delta y_{t-j}-\sum_{l=1}^{m} \rho_{l} \varepsilon_{t-l}(\phi, \boldsymbol{\theta}),
$$

where $\varepsilon_{t}(\phi, \boldsymbol{\theta})=0$ for $t<1$. Then, the weighted score functions denoted by $\mathbf{Z}_{t}(\phi, \boldsymbol{\theta})$ can also be constructed as those in (4.2) and (4.4). Furthermore, if $n^{-1 / 2} \sum_{t=1}^{n} \mathbf{Z}_{t}\left(1, \boldsymbol{\theta}_{0}\right)$ is asymptotically normal and $\partial^{k} \mathbf{Z}_{t}(\phi, \boldsymbol{\theta}) / \partial \boldsymbol{\theta}^{k}$ is uniformly bounded for $k=1,2,3$, the asymptotically chi-squared property can be derived by a similar proof procedure for Theorem 4.1-4.2; see the Supplementary for technical details. Therefore, our empirical likelihood methods are feasible to many structures of unit root models.

Remark 4.2. As suggested by one of the referees, we consider the unit root testing 
problem when the noise is a linear process, namely,

$$
y_{t}=\phi y_{t-1}+u_{t}=\phi y_{t-1}+\varepsilon_{t}+\sum_{l=1}^{\infty} \rho_{l} \varepsilon_{t-l} .
$$

Different from all the foregoing models, infinite number of parameters $\left\{\rho_{1}, \rho_{2}, \cdots\right\}$ are involved in (4.6). Thus, it is not feasible to use the recursive definition for $\varepsilon_{t}(\phi, \boldsymbol{\theta})$ as (4.5), where $m$ is finite. One possible method is to utilize the basic idea in the ADF test, as seen, for example, in Zhang and Chan (2020). Specifically, under $H_{0}$, the model (4.6) can be rewritten as

$$
y_{t}=\phi y_{t-1}+\sum_{j=1}^{k} \beta_{j} \Delta y_{t-j}+\varepsilon_{t}+\rho_{t, k}
$$

where $\left(1-\sum_{j=1}^{\infty} \beta_{j} z^{j}\right)$ is the inverse function of $\left(1+\sum_{l=1}^{\infty} \rho_{l} z^{l}\right)$ and $\rho_{t, k}=\sum_{j=k+1}^{\infty} \beta_{j} u_{t-j}$.

Then, we define $\varepsilon_{t}(\phi, \boldsymbol{\theta})=y_{t}-\phi y_{t-1}-\sum_{j=1}^{k} \beta_{j} \Delta y_{t-j}$ with $\boldsymbol{\theta}=\left(\beta_{1}, \cdots, \beta_{k}\right)^{\prime}$. Note that $\varepsilon_{t}\left(1, \boldsymbol{\theta}_{0}\right)=\varepsilon_{t}+\rho_{t, k}$ and thus it is necessary to select an appropriate $k \rightarrow \infty$ as $n \rightarrow \infty$. Nevertheless, this procedure involves high-dimensional empirical likelihood, which is substantially different from the methodology in fixed dimensional case. Therefore, we leave this problem for the other independent project.

\section{Simulation Studies}

To examine the finite sample behavior of the proposed unit-root tests, we focus on the model (1.1) with $\phi=1$ corresponding to the null hypothesis (i.e., unit-root) and $\phi \in\{0.95,0.9,0.85\}$ corresponding to the alternative (i.e., stationary). In all 
simulations, we take 1000 replications for each case and the results are reported at the significant level $5 \%$.

\subsection{The GARCH-type noises}

In this section, we consider the GARCH-type noises as follows:

$$
\begin{gathered}
\varepsilon_{t}=\eta_{t} h_{t}, h_{t}^{2}=\omega+\left[\beta_{1}+\alpha_{1} \eta_{t-1}^{2}+\gamma \eta_{t-1}^{2} 1_{\left(\eta_{t-1}<0\right)}\right] h_{t-1}^{2}, \\
\varepsilon_{t}=\eta_{t} h_{t}, h_{t}^{2}=\omega+\left\{\beta_{1}+\alpha_{1}\left[1-2 \gamma \operatorname{sign}\left(\eta_{t-1}\right)+\gamma^{2}\right] \eta_{t-1}^{2}\right\} h_{t-1}^{2},
\end{gathered}
$$

where $\omega=0.1$ and $\gamma=0.1$. Let $\boldsymbol{\theta}=\left(\beta_{1}, \alpha_{1}\right)$ be the unknown parameters and $\alpha$ be the tail index of $\varepsilon_{t}$. By Remark 2.1, the following heavy-tailed cases are considered:

1. For $\alpha \in(1,2)$, we uniformly take (i) $\boldsymbol{\theta}=(0.6,0.4)$ with $\eta_{t}$ being $N(0,1)$; (ii) $\boldsymbol{\theta}=(0.5,0.3)$ with $\eta_{t}$ being Laplace $(0,1)$ distribution; (iii) $\boldsymbol{\theta}=(0.7,0.1)$ with $\eta_{t}$ being $t_{3}$ distribution; (iv) $\boldsymbol{\theta}=(0.5,0.1)$ with $\eta_{t}$ being $t_{2}$ distribution.

2. For $\alpha \in(0,1)$, we uniformly take (i) $\boldsymbol{\theta}=(0.6,0.5)$ with $\eta_{t}$ being $N(0,1)$; (ii) $\boldsymbol{\theta}=(0.5,0.4)$ with $\eta_{t}$ being Laplace $(0,1)$ distribution; (iii) $\boldsymbol{\theta}=(0.65,0.1)$ with $\eta_{t}$ being $t_{2}$ distribution; (iv) $\boldsymbol{\theta}=(0.35,0.1)$ with $\eta_{t}$ being Cauchy distribution.

For comparison, we also implement the empirical likelihood-based test (ELT) proposed by Huang et al. (2020) with the order $m=2$ (see Remark 3.1).

Tables 1-2 report the size and power of the tests with $\alpha \in(1,2)$ when $n=100$ and $n=300$, respectively. Under the null hypothesis $(\phi=1)$, it is apparent that 
the proposed test $\tilde{T}_{n}$ is always under-sized. In particular, the size of $\tilde{T}_{n}$ is only 0.016 in model (5.2) when $\eta_{t} \sim N(0,1)$ and $n=100$. Instead, the proposed empirical likelihood tests $l(1)$ and $l^{a}(1)$ present a satisfactory size performance, whereas the ELT is oversized and the size distortion becomes severer as the tail of $\eta_{t}$ becomes heavier. Besides, we can see that all the empirical likelihood tests are improved in power when the sample size increases from 100 to 300. In contrast, the proposed tests $l(1)$ and $l^{a}(1)$ are more powerful than the ELT in almost all cases except for $\eta_{t} \sim N(0,1)$, where the ELT is better but the gap is acceptable. Similar phenomena can also be observed in Tables 3-4, which summarize the associated simulation results for $\alpha \in(0,1)$.

For the extremely heavy-tailed noise, it is seen from Tables 3-4 that the size and power performances of $l(1)$ and $l^{a}(1)$ are quite robust with a stable size and a rational power. However, when $\eta_{t}$ follows the Cauchy distribution, the ELT can suffer from a serious size distortion and have a severe loss in power, even for large sample size. Therefore, the existing ELT is sensitive to the tail of $\eta_{t}$ and may not be used in unit-root testing when the tail index is unknown. In summary, our proposed empirical likelihood tests are efficient and powerful to detect a possible unit root, especially for models with heavy-tailed innovations.

\subsection{The i.i.d. noises}

We now conduct a simulation study to illustrate that the proposed tests are still 
Table 1: Size and power of the unit-root tests $(\times 100)$ with $\alpha \in(1,2)$ and $n=100$

\begin{tabular}{|c|c|c|c|c|c|c|c|c|c|}
\hline \multirow[b]{2}{*}{$\eta_{t} \sim$} & \multirow[b]{2}{*}{$\phi$} & \multicolumn{4}{|c|}{$\varepsilon_{t} \sim \operatorname{model}(5.1)$} & \multicolumn{4}{|c|}{$\varepsilon_{t} \sim \operatorname{model}(5.2)$} \\
\hline & & $\tilde{T}_{n}$ & $l(1)$ & $l^{a}(1)$ & ELT & $\tilde{T}_{n}$ & $l(1)$ & $l^{a}(1)$ & ELT \\
\hline \multirow[t]{4}{*}{$\mathrm{N}(0,1)$} & 1.00 & 2.4 & 4.8 & 4.7 & 5.7 & 1.6 & 4.5 & 4.9 & 6.7 \\
\hline & 0.95 & 19.3 & 19.8 & 17.3 & 22.6 & 17.6 & 19.5 & 16.7 & 23.5 \\
\hline & 0.90 & 34.1 & 36.0 & 33.9 & 47.0 & 32.6 & 39.0 & 37.0 & 49.3 \\
\hline & 0.85 & 54.9 & 58.1 & 55.9 & 68.4 & 51.5 & 60.2 & 57.5 & 72.8 \\
\hline \multirow[t]{4}{*}{ Laplace } & 1.00 & 3.4 & 5.3 & 4.8 & 6.5 & 2.9 & 4.9 & 4.8 & 6.2 \\
\hline & 0.95 & 42.7 & 39.0 & 36.6 & 28.9 & 38.2 & 36.1 & 34.0 & 25.5 \\
\hline & 0.90 & 64.1 & 62.3 & 59.9 & 47.5 & 59.7 & 60.1 & 57.0 & 48.7 \\
\hline & 0.85 & 77.1 & 78.7 & 78.7 & 58.9 & 77.9 & 79.4 & 78.1 & 63.9 \\
\hline \multirow[t]{4}{*}{$t_{3}$} & 1.00 & 2.8 & 5.1 & 4.7 & 7.3 & 2.8 & 4.2 & 5.0 & 7.1 \\
\hline & 0.95 & 35.2 & 28.6 & 26.9 & 21.5 & 27.9 & 26.9 & 25.2 & 23.0 \\
\hline & 0.90 & 57.4 & 53.0 & 50.5 & 39.3 & 58.1 & 60.5 & 58.2 & 40.3 \\
\hline & 0.85 & 77.9 & 76.1 & 73.9 & 52.1 & 80.8 & 84.7 & 82.8 & 55.9 \\
\hline \multirow[t]{4}{*}{$t_{2}$} & 1.00 & 4.3 & 5.5 & 4.9 & 8.8 & 3.6 & 5.6 & 5.1 & 8.8 \\
\hline & 0.95 & 54.4 & 51.1 & 48.8 & 28.0 & 54.2 & 52.5 & 50.1 & 25.7 \\
\hline & 0.90 & 80.2 & 80.3 & 77.9 & 41.7 & 81.9 & 82.7 & 81.4 & 43.4 \\
\hline & 0.85 & 91.5 & 91.5 & 90.3 & 51.0 & 92.0 & 94.6 & 94.2 & 49.2 \\
\hline
\end{tabular}

Table 2: Size and power of the unit-root tests $(\times 100)$ with $\alpha \in(1,2)$ and $n=300$

\begin{tabular}{|c|c|c|c|c|c|c|c|c|c|}
\hline \multirow[b]{2}{*}{$\eta_{t} \sim$} & \multirow[b]{2}{*}{$\phi$} & \multicolumn{4}{|c|}{$\varepsilon_{t} \sim \operatorname{model}(5.1)$} & \multicolumn{4}{|c|}{$\varepsilon_{t} \sim \operatorname{model}(5.2)$} \\
\hline & & $\tilde{T}_{n}$ & $l(1)$ & $l^{a}(1)$ & ELT & $\tilde{T}_{n}$ & $l(1)$ & $l^{a}(1)$ & ELT \\
\hline \multirow[t]{4}{*}{$\mathrm{N}(0,1)$} & 1.00 & 3.6 & 5.5 & 5.3 & 5.7 & 3.6 & 4.6 & 4.7 & 5.4 \\
\hline & 0.95 & 71.6 & 66.7 & 65.6 & 76.6 & 73.1 & 68.4 & 67.0 & 75.0 \\
\hline & 0.90 & 94.2 & 92.3 & 91.6 & 96.6 & 96.1 & 96.5 & 95.4 & 97.6 \\
\hline & 0.85 & 98.9 & 98.5 & 98.3 & 99.3 & 99.5 & 99.5 & 99.4 & 99.8 \\
\hline \multirow[t]{4}{*}{ Laplace } & 1.00 & 3.7 & 4.9 & 4.7 & 6.4 & 3.3 & 4.3 & 4.9 & 5.7 \\
\hline & 0.95 & 96.3 & 94.1 & 93.8 & 79.0 & 93.9 & 92.8 & 92.6 & 80.4 \\
\hline & 0.90 & 99.6 & 99.9 & 99.6 & 92.8 & 99.7 & 99.9 & 99.7 & 94.7 \\
\hline & 0.85 & 100 & 100 & 100 & 94.8 & 100 & 100 & 100 & 98.2 \\
\hline \multirow[t]{4}{*}{$t_{3}$} & 1.00 & 4.3 & 5.4 & 5.2 & 6.5 & 3.9 & 5.3 & 5.0 & 6.9 \\
\hline & 0.95 & 91.4 & 86.3 & 85.5 & 84.4 & 91.9 & 88.8 & 88.9 & 59.9 \\
\hline & 0.90 & 99.4 & 99.1 & 99.1 & 76.4 & 99.7 & 99.7 & 99.7 & 83.7 \\
\hline & 0.85 & 100 & 100 & 100 & 89.3 & 100 & 100 & 100 & 91.9 \\
\hline \multirow[t]{4}{*}{$t_{2}$} & 1.00 & 3.6 & 4.7 & 4.9 & 8.0 & 3.5 & 4.4 & 4.8 & 7.7 \\
\hline & 0.95 & 99.2 & 98.7 & 98.5 & 59.3 & 99.1 & 98.8 & 98.8 & 59.4 \\
\hline & 0.90 & 100 & 100 & 100 & 73.1 & 100 & 100 & 100 & 75.3 \\
\hline & 0.85 & 100 & 100 & 100 & 79.8 & 100 & 100 & 100 & 82.7 \\
\hline
\end{tabular}


Table 3: Size and power of the unit-root tests $(\times 100)$ with $\alpha \in(0,1)$ and $n=100$

\begin{tabular}{|c|c|c|c|c|c|c|c|c|c|}
\hline \multirow[b]{2}{*}{$\eta_{t} \sim$} & \multirow[b]{2}{*}{$\phi$} & \multicolumn{4}{|c|}{$\varepsilon_{t} \sim \operatorname{model}(5.1)$} & \multicolumn{4}{|c|}{$\varepsilon_{t} \sim \operatorname{model}(5.2)$} \\
\hline & & $\tilde{T}_{n}$ & $l(1)$ & $l^{a}(1)$ & ELT & $\tilde{T}_{n}$ & $l(1)$ & $l^{a}(1)$ & ELT \\
\hline \multirow{4}{*}{$\mathrm{N}(0,1)$} & 1.00 & 3.9 & 5.0 & 4.8 & 5.6 & 3.2 & 5.0 & 4.7 & 6.1 \\
\hline & 0.95 & 21.9 & 21.6 & 19.2 & 23.5 & 22.4 & 19.4 & 17.4 & 21.9 \\
\hline & 0.90 & 37.9 & 31.4 & 30.0 & 39.7 & 36.9 & 34.0 & 31.1 & 44.5 \\
\hline & 0.85 & 49.0 & 43.7 & 41.2 & 55.8 & 53.5 & 51.3 & 49.4 & 60.4 \\
\hline \multirow[t]{4}{*}{ Laplace } & 1.00 & 4.1 & 5.5 & 4.7 & 6.3 & 3.3 & 4.6 & 4.9 & 5.9 \\
\hline & 0.95 & 47.8 & 41.1 & 40.7 & 29.7 & 48.7 & 41.7 & 41.2 & 29.1 \\
\hline & 0.90 & 66.8 & 58.8 & 56.7 & 43.8 & 66.9 & 61.0 & 58.2 & 45.3 \\
\hline & 0.85 & 78.0 & 72.9 & 70.9 & 51.4 & 78.5 & 75.7 & 74.6 & 58.1 \\
\hline \multirow[t]{4}{*}{$t_{2}$} & 1.00 & 3.3 & 4.6 & 4.5 & 9.1 & 3.6 & 4.9 & 4.8 & 10.3 \\
\hline & 0.95 & 51.9 & 41.5 & 41.8 & 26.5 & 51.7 & 44.3 & 43.7 & 24.0 \\
\hline & 0.90 & 74.9 & 67.4 & 65.3 & 35.3 & 77.9 & 73.4 & 70.7 & 37.2 \\
\hline & 0.85 & 82.9 & 78.5 & 76.7 & 41.4 & 91.6 & 90.2 & 89.1 & 45.4 \\
\hline \multirow[t]{4}{*}{ Cauchy } & 1.00 & 4.2 & 4.8 & 4.5 & 21.6 & 4.6 & 5.2 & 4.7 & 19.5 \\
\hline & 0.95 & 93.4 & 89.3 & 88.7 & 33.8 & 95.0 & 92.9 & 92.5 & 36.1 \\
\hline & 0.90 & 98.2 & 96.2 & 95.8 & 35.5 & 99.2 & 98.1 & 97.8 & 35.3 \\
\hline & 0.85 & 99.0 & 97.5 & 97.5 & 38.8 & 99.3 & 98.9 & 98.9 & 36.3 \\
\hline
\end{tabular}

Table 4: Size and power of the unit-root tests $(\times 100)$ with $\alpha \in(0,1)$ and $n=300$

\begin{tabular}{|c|c|c|c|c|c|c|c|c|c|}
\hline \multirow[b]{2}{*}{$\eta_{t} \sim$} & \multirow[b]{2}{*}{$\phi$} & \multicolumn{4}{|c|}{$\varepsilon_{t} \sim \operatorname{model}(5.1)$} & \multicolumn{4}{|c|}{$\varepsilon_{t} \sim \operatorname{model}(5.2)$} \\
\hline & & $\tilde{T}_{n}$ & $l(1)$ & $l^{a}(1)$ & ELT & $\tilde{T}_{n}$ & $l(1)$ & $l^{a}(1)$ & ELT \\
\hline \multirow[t]{4}{*}{$\mathrm{N}(0,1)$} & 1.00 & 4.4 & 4.8 & 4.8 & 7.2 & 4.7 & 4.9 & 4.8 & 6.6 \\
\hline & 0.95 & 67.2 & 58.0 & 57.3 & 65.8 & 74.2 & 65.4 & 64.6 & 71.1 \\
\hline & 0.90 & 85.6 & 78.4 & 77.3 & 84.4 & 90.6 & 85.8 & 85.4 & 90.1 \\
\hline & 0.85 & 96.0 & 91.7 & 91.6 & 94.2 & 96.9 & 95.7 & 95.5 & 96.2 \\
\hline \multirow[t]{4}{*}{ Laplace } & 1.00 & 5.8 & 5.1 & 5.0 & 6.6 & 4.8 & 4.9 & 4.7 & 6.5 \\
\hline & 0.95 & 97.3 & 94.0 & 94.7 & 74.7 & 96.6 & 94.7 & 94.2 & 78.1 \\
\hline & 0.90 & 99.4 & 98.9 & 98.9 & 84.0 & 99.8 & 99.6 & 99.6 & 89.3 \\
\hline & 0.85 & 99.8 & 99.8 & 99.8 & 89.1 & 99.6 & 99.6 & 99.5 & 94.3 \\
\hline \multirow[t]{4}{*}{$t_{2}$} & 1.00 & 4.7 & 5.3 & 5.2 & 9.2 & 4.2 & 4.6 & 4.7 & 7.6 \\
\hline & 0.95 & 98.1 & 95.9 & 95.7 & 49.8 & 98.7 & 97.6 & 97.4 & 50.5 \\
\hline & 0.90 & 99.8 & 99.6 & 99.6 & 60.1 & 100 & 100 & 100 & 69.9 \\
\hline & 0.85 & 100 & 100 & 100 & 66.8 & 100 & 100 & 100 & 72.9 \\
\hline \multirow[t]{4}{*}{ Cauchy } & 1.00 & 4.2 & 5.4 & 5.2 & 20.5 & 4.4 & 4.5 & 4.6 & 22.6 \\
\hline & 0.95 & 100 & 100 & 100 & 42.3 & 100 & 100 & 100 & 42.3 \\
\hline & 0.90 & 100 & 100 & 100 & 39.1 & 100 & 100 & 100 & 39.9 \\
\hline & 0.85 & 100 & 100 & 100 & 35.4 & 100 & 100 & 100 & 37.7 \\
\hline
\end{tabular}


valid when $\varepsilon_{t}$ are i.i.d. (i.e., $h_{t}=1$ ) with an infinite variance. To be precise, the noise $\varepsilon_{t}$ is generated from the model:

$$
\varepsilon_{t}=\left|\eta_{t}\right|^{1 / \alpha} \operatorname{sign}\left(\eta_{t}\right)
$$

where $\eta_{t} \sim$ Cauchy distribution and $\alpha$ is the tail index. For comparison, we also consider two common ADF-type tests:

$$
R_{n, \kappa}=\frac{(n-k)(\hat{\phi}-1)}{1-\sum_{j=1}^{k} \hat{\beta}_{j}}, \quad Q_{n, \kappa}=\frac{\hat{\phi}-1}{s(\hat{\phi})}
$$

where $\left(\hat{\phi}, \hat{\beta}_{1}, \cdots, \hat{\beta}_{k}\right)$ is the LSE of regression parameters in model $(4.7), s(\hat{\phi})$ is the usual standard error of $\hat{\phi}$, and $k=\left[\kappa(n / 100)^{1 / 4}\right]$ is the selected lag length with $\kappa=4$ and 12. To implement these two tests in heavy-tailed cases, we take the wild sieve bootstrap method proposed by Cavaliere, Georgiev and Taylor (2018) with bootstrap size $b=1000$ and denote the associated tests as $R_{n, \kappa}^{b}$ and $Q_{n, \kappa}^{b}$. The simulation results are presented in Tables 5-6.

It is seen from Tables $5-6$ that the proposed empirical likelihood tests $l(1)$ and $l^{a}(1)$ outperform all competitors in power performance with a stable size for nearly all cases, while the wild bootstrap tests are sensitive to the selected lag length $\kappa$ where a smaller $\kappa$ means a higher power, which is consistent with that in Cavaliere, Georgiev and Taylor (2018). In addition, it is interesting to see that the proposed tests become more powerful when the tail of the noise becomes heavier. 
Table 5: Size and power of the unit-root tests $(\times 100)$ for i.i.d. noise when $n=100$

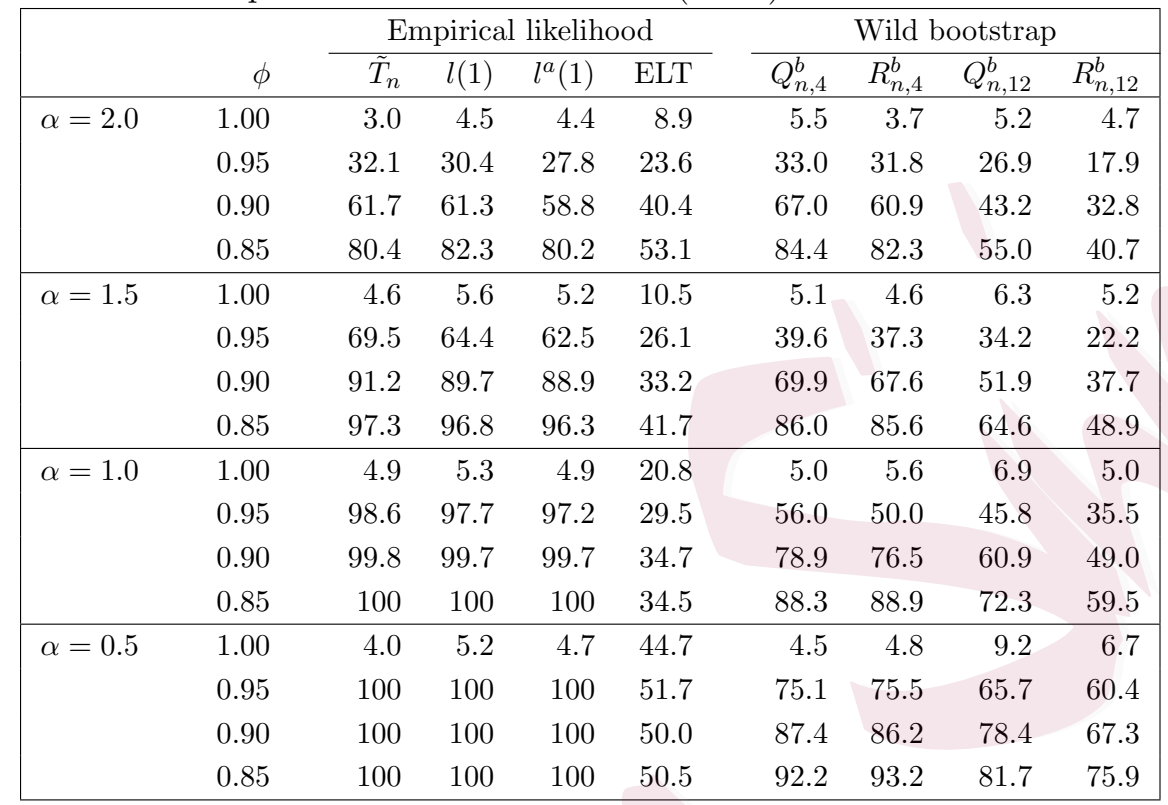

Table 6: Size and power of the unit-root tests $(\times 100)$ for i.i.d. noise when $n=300$

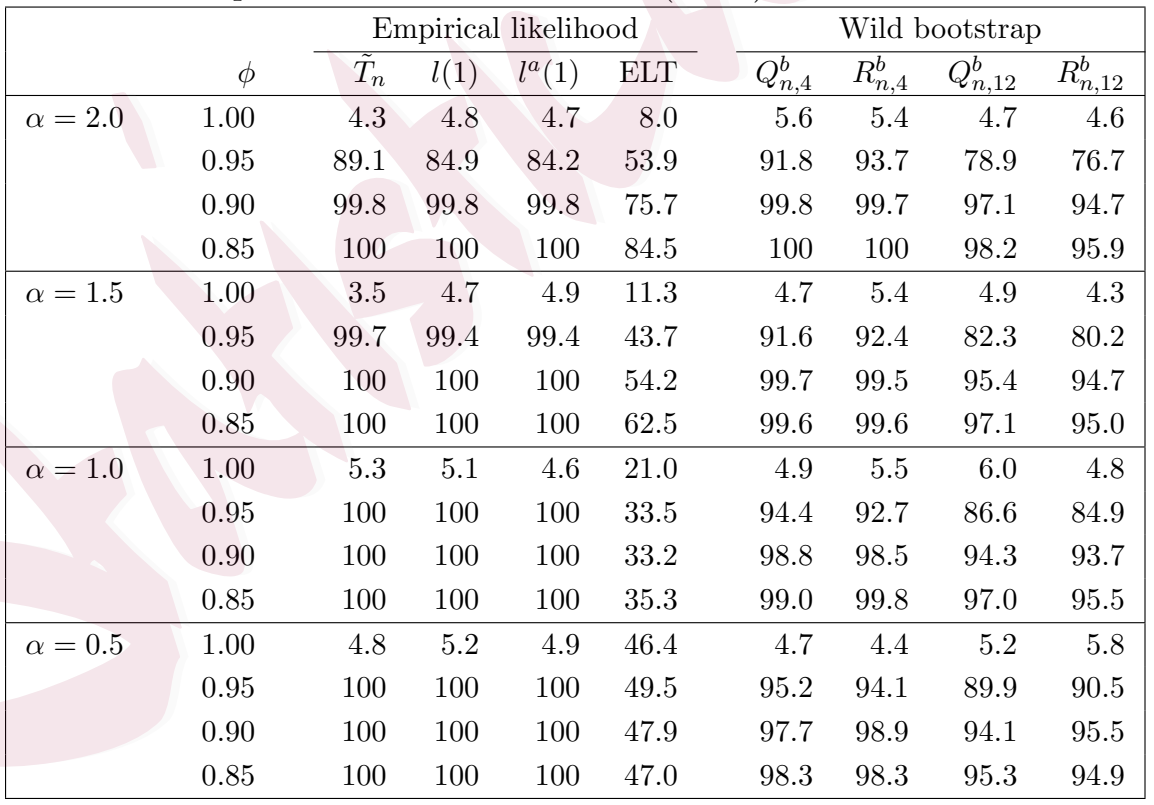


REFERENCES30

\section{Supplementary Material}

The Supplementary Material contains the technical proofs for the results in Sections $2-4$, and the simulation results for confidence interval estimation under the alternative.

\section{Acknowledgments}

The author would like to thank the Editor, Associate Editor and two anonymous reviewers for their insightful comments and suggestions that have substantially improved the content of this paper. I also would like to thank Professor Shiqing Ling for his guidance on the GARCH process and Professor Deyuan Li for helpful discussions on empirical likelihood method. Rui She's research was supported by Fundamental Research Funds for the Central Universities (Nos. JBK1806002 and JBK190905) of China.

\section{References}

Avram, F. and Taqqu, M. (1992). Weak convergence of sums of moving averages in the $\alpha$-stable domain of attraction. Ann. Probab. 20, 483-503.

Billingsley, P. (1999). Convergence of Probability Measures, 2nd Ed. Wiley, New York.

Caner, M. (1998). Tests for cointegration with infinite variance errors. J. Econom. 86, 155-175.

Cavaliere, G., Georgiev, I. and Taylor, A. M. R. (2018). Unit root inference for non-stationary linear processes driven by infinite variance innovations. Econom. Theory 34(02), 302-348. 
Chan, N.H. (2009). Time series with roots on or near the unit circle. In Handbook of Financial Time Series, 695-707. Springer-Verlag, New York.

Chan, N.H., Li, D. and Peng, L. (2012). Toward a unified interval estimation of autoregressions. Econom. Theory 28, 705-717.

Chan, N. H. and Tran, L. T. (1989). On the first-order autoregressive process with infinite variance. Econom. Theory 5, 354-362.

Chan, N. H. and Zhang, R.M. (2010). Inference for unit-root models with infinite variance GARCH errors. Statistica Sinica, 20, 1363-1393.

Chen, J., Variyath, A. M. and Abraham, B. (2008). Adjusted empirical likelihood and its properties. J. Comput. Graph. Stat. 17, 426-443.

Dickey, D.A. and Fuller, W.A. (1979). Distribution of the estimators for autoregressive time series with a unit root. J. Am. Stat. Assoc. 74, 427-431.

Engle, R.F. (1990). Discussion: Stock market volatility and the crash of 1987 . Rev. Financ. Stud. 3, 103-106.

Evans, G.B.A. and Savin, N.E. (1981). Testing for Unit Roots:1. Econometrica, 49, 753-782.

Fornari, F. and Mele, A. (1997). Sign- and Volatility-switching ARCH models: theory and applications to international stock arkets. J. Appl. Econom. 12, 1779-1801.

Francq, C. and Zakoïan, J. (2013). Estimating the marginal law of a time series with applications to heavy-tailed distributions. J. Bus. Econ. Stat. 31, 412-425. 
Glosten, L. R., Jagannathan, R. and Runkle, D. (1993). On the relation between the expected value and the volatility of the nominal excess return on stocks. J. Finance 48, 1779-1801.

Horváth, L. and Kokoszka, P.S. (2003). A bootstrap approximation to a unit root test statistic for heavy tailed observations. Stat. Probab. Lett. 62, 163-173.

Huang, H.T., Leng, X., Liu, X.H. and Peng, L. (2020). Unified inference for an AR process regardless of finite or infinite variance GARCH errors. J. Financ. Econom. 18(2), 425-470.

Jakubowski, A. (1997). A non-Skorohod topology on the Skorohod space. Electron. J. Probab. 2, 1-21.

Knight, K. (1991). Limit theory for M-estimates in an integrated infinite variance process. Econom. Theory 7, 200-212.

Koedijk, K.G. and Kool, C.J.M. (1992). Tail estimates of East European exchange rates. J. Bus. Econ. Stat. 10, 83-96.

Li, D., Chan, N. H. and Peng, L. (2014). Empirical likelihood test for causality for bivariate AR(1) processes. Econom. Theory 30, 357-371.

Moreno M. and Romo J. (2012). Unit root bootstrap tests under infinite variance. J. Time Ser. Anal. 33, $32-47$.

Owen, A.B. (2001). Empirical Likelihood, New York: Chapman \& Hall/CRC.

Phillips, P.C.B. (1987). Time series regression with a unit root. Econometrica 55, 277-301.

Phillips, P.C.B. (1990). Time series regression with a unit root and infinite-variance errors. Econom. Theory 6, 44-62. 
Qin, J. and Lawless, J. (1994). Empirical likelihood and general estimation equations. Ann. Statist. 22, 300-325.

Rachev, S.T. (2003). Handbook of Heavy Tailed Distributions in Finance. Elsevier/North-Holland.

Resnick, S. I. (1997). Heavy tail modeling and teletraffic data. (With discussion and rejoinder by author). Ann. Statist. 25, 1805-1869.

Samarakoon, D.M.M. and Knight, K. (2009). A note on unit root tests with infinite variance noise. Econom. Rev. 28(4), 314-334.

Schwert, G.W. (1989). Why does stock market volatility change over time? J. Finance 45, 1129-1155.

Sentana, E. (1995). Quadratic ARCH models. Rev. Econ. Stud. 62, 639-661.

She, R. and Ling, S. (2020). Inference in heavy-tailed vector error correction models. J. Econom. 214, 433-450.

Shin, D.W. and So, B.S. (1999). New tests for unit roots in autoregressive processes with possibly infinite variance errors. Stat. Probab. Lett. 44, 387-397.

Taylor, S. (1986). Modelling Financial Time Series, New York: Wiley.

Zakoïan, J. M. (1994). Threshold heteroskedastic models. J. Econ. Dyn. Control 18, 931-955.

Zhang, R.M. and Chan, N. H. (2020). Nonstationary linear processes with infinite variance GARCH errors. Econom. Theory, 0, 1-34.

Zhang, R. M. and Ling, S. (2015) Asymptotic inference for AR models with heavy-tailed G-GARCH noises. Econom. Theory, 31, 880-890. 
Zhang, R.M., Sin, C.Y. and Ling, S. (2015). On functional limits of short- and long-memory linear processes with GARCH(1,1) noises. Stoch. Process. Their Appl. 125, 482-512.

Zheng, M. and Yu, W. (2013). Empirical likelihood method for multivariate Cox regressions. Comput. Stat. 28, 1241-1267.

Rui She

Center of Statistical Research, School of Statistics, Southwestern University of Finance and Economics,

Chengdu, China.

E-mail: rshe@swufe.edu.cn 